\title{
Technè
}

La science au service de l'histoire de l'art et de la préservation des biens culturels

44 | 2016

Archives de l'humanité : les restes humains patrimonialisés

\section{Les restes humains « patrimonialisés » et la loi}

Granting human remains "heritage status" and the law

\section{Marie Cornu}

\section{(2) OpenEdition}

1 Journals

\section{Édition électronique}

URL : http://journals.openedition.org/techne/909

DOI : 10.4000/techne.909

ISSN : 2534-5168

Éditeur

C2RMF

\section{Édition imprimée}

Date de publication : 1 novembre 2016

Pagination : 8-13

ISBN : 978-2-7118-6339-6

ISSN : 1254-7867

\section{Référence électronique}

Marie Cornu, « Les restes humains « patrimonialisés » et la loi », Technè [En ligne], 44 | 2016, mis en ligne le 19 décembre 2019, consulté le 10 décembre 2020. URL : http://journals.openedition.org/ techne/909; DOI : https://doi.org/10.4000/techne.909

\section{(c) (i) (9)}

La revue Technè. La science au service de l'histoire de l'art et de la préservation des biens culturels est mise à disposition selon les termes de la Licence Creative Commons Attribution - Pas d'Utilisation Commerciale - Pas de Modification 4.0 International. 


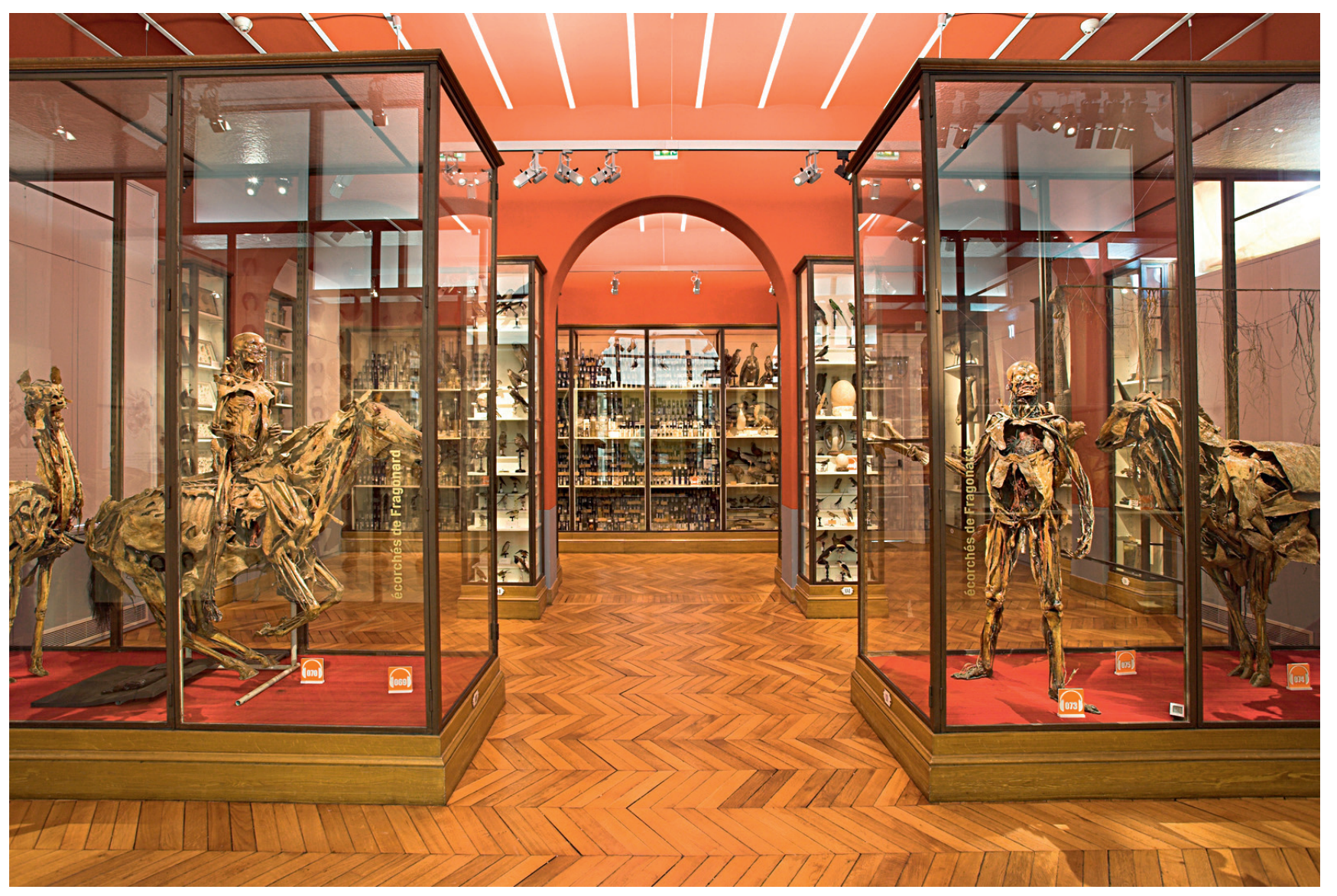

Fig. 1. Cabinet de curiosités du musée Fragonard de l'École nationale vétérinaire d'Alfort, à Maisons-Alfort. À gauche, le cavalier anatomisé d'Honoré Fragonard.

(c) Musée Fragonard de l'École nationale vétérinaire d'Alfort/C. Degueurce. 
Marie Cornu
Les restes humains «patrimonialisés » et la loi
Granting human remains "heritage status" and the law
Résumé. Le processus de patrimonialisation du corps humain soulève des questions complexes sur le double terrain du droit et de l'éthique, qui se rapportent tant au statut des restes humains qu'à leur traitement juridique et scientifique. Si le droit les considère comme des "choses " indisponibles, cette condition n'exclut pas leur conservation dans une institution patrimoniale, dans des termes qui relèvent davantage de la déontologie que du droit.

Mots-clés. Principe de dignité, statut juridique de la dépouille, collection publique, domaine public, restitution, têtes maories, déontologie.

\author{
Abstract. The process of incorporating the human body into \\ cultural heritage raises complex questions in the twofold field of \\ law and ethics, which relates to both the status of human remains \\ and their legal and scientific treatment. While the law regards \\ them as unavailable "things", this condition does not exclude their \\ conservation in a heritage institution, in terms more relevant to \\ deontology than law. \\ Keywords. Principle of human dignity, legal status of human \\ remains, public collection, public domain, restitution, Maori \\ heads, deontology.
}

La dépouille mortelle a pour destination l'inhumation ou, à l'état de cendres, la conservation ou la dispersion dans des modalités qu'encadre la loi funéraire récemment révisée ${ }^{1}$. Leur condition juridique n'est cependant pas entièrement déterminée par ces textes. C'est que le corps humain est doublement un objet d'études pour la science et un objet patrimonial, entré au musée sous des formes multiples. Comment traiter juridiquement ces sortes de choses ${ }^{2}$ ? Peut-on les «patrimonialiser» et dans quels termes ? La question recèle en réalité une forte ambiguïté, en raison du fait que le terme de patrimoine peut être entendu de plusieurs façons. Publicisée en France par MM. Aubry et Rau, dans le sillage d'un auteur allemand, Zachariae, la théorie du patrimoine au sens du droit civil - premier conceptuellement à paraître - renvoie aux biens et obligations d'une personne. Positivement, la patrimonialité, reçue dans un sens économique, peut être définie comme la capacité d'une chose ou d'un droit à intégrer la masse de ses actifs. Mais la notion de patrimoine, plus récemment réappropriée par le droit public, endosse un autre sens. Il désigne selon l'article L. 1 du Code du patrimoine «l'ensemble des biens, immobiliers ou mobiliers, relevant de la propriété publique ou privée, qui présentent un intérêt historique, artistique, archéologique, esthétique, scientifique ou technique... ». C'est cette fois-ci le patrimoine culturel, composé notamment des monuments historiques, des collections des musées, des sites et objets archéologiques, des archives, etc ${ }^{3}$. Nous nous concentrons sur cette réalité, laissant de côté le statut des collections scientifiques récentes encadrées notamment par le Code de la santé publique. Sous cette perspective, se demander dans quels termes «patrimonialiser» les restes humains au sens du Code du patrimoine nécessite en réalité de sonder ces deux versants de la patrimonialité économique et culturelle.

\section{La patrimonialité au sens économique du terme : l'indisponibilité relative du corps décédé}

Le corps humain, au décès de la personne, peut-il être considéré comme un élément du patrimoine ? L'article 16-1 du Code civil issu de la loi n 94-653 du 29 juillet 1994 dispose que « chacun a droit au respect de son corps. Le corps humain est inviolable. Le corps humain, ses éléments et ses produits ne peuvent faire l'objet d'un droit patrimonial ». La règle d'indisponibilité perdure après la mort, précision introduite à l'article 16-1-1 du Code civil par la loi n 2008-1350 du 19 décembre 2008, cependant que le corps humain change de statut. De son vivant, c'est la personne qui, juridiquement, reçoit protection. À sa mort, le respect du corps emporte comme conséquence principale la prohibition d'un commerce lucratif, ce qui n'exclut cependant pas toute patrimonialité. Le corps décédé peut être approprié. 


\section{La possibilité d'un droit de propriété}

La nature spéciale des restes humains ne fait aucun doute sur le plan juridique. Un certain nombre de textes leur réserve un sort particulier, inspirés par des motifs divers : respect dû au corps et principe de dignité, encadrement des recherches biomédicales, législation funéraire, sort des dépouilles militaires... Dans ce corpus dispersé, la doctrine juridique éprouve un certain embarras dans l'exercice de qualification. À l'intérieur de quelle catégorie ranger la dépouille ? Ce n'est pas une personne au sens où le droit l'entend, dès lors que la personnalité juridique cesse avec la mort. Si le corps humain rejoint le monde des choses ${ }^{4}$, il n'est évidemment pas une chose comme les autres, qui pourrait être « estimable, évaluable, échangeable ${ }^{5}$ ». La plupart des règles applicables à cette chose spéciale, profondément marquée par l'empreinte de la personne, expriment l'idée d'une indisponibilité dans des contours et modalités variables. S'agit-il d'une chose sans maître, d'une chose commune, d'une chose hors commerce ${ }^{6}$ ? Peut-on imaginer qu'elle puisse être appropriée ? La loi et la jurisprudence semblent bien l'admettre, même si la propriété apparaît comme une qualification par défaut, en l'absence d'autres figures disponibles. Lorsque, par exemple, le juge punit le vol de cadavre, il sanctionne une atteinte portée à la propriété d'autrui ${ }^{7}$. Sur un autre registre, dans un certain nombre de décisions, le juge, pour déterminer, parfois départager, les droits de la famille sur la dépouille, en appelle à la figure de la copropriété familiale ${ }^{8}$, forme particulière de propriété évidemment non assimilable à la propriété privée au sens de l'article 544 du Code civil ${ }^{9}$. On pourrait encore évoquer la découverte de l'homme de glace qui a donné lieu à un droit d'invention, forme d'attribution de la propriété d'un bien en tout ou partie, au bénéfice de ceux qui en ont fait la découverte.

Cette question de l'appropriation des restes a également ressurgi dans des termes propres avec le contentieux de la Ville de Rouen entreprenant la restitution auprès de la communauté maorie d'une tête de guerrier entrée à la fin du $\mathrm{XIX}^{\mathrm{e}}$ siècle dans ses collections. Cette fois-ci, la discussion se noue autour de la présence de cet élément du corps humain dans une collection publique. La Ville de Rouen, au soutien de sa démarche, estime « qu'en application de l'article 16-1 du Code civil, des restes humains, dont le retour est réclamé pour être inhumés, ne peuvent en aucune manière être considérés comme susceptibles d'appropriation privée comme publique ». Le Code civil ne proscrirait pas seulement, selon le moyen, «l'appropriation à des fins mercantiles, mais toute forme d'appropriation privée ou publique ». L'impossibilité d'une propriété aurait évidemment fait échec en ce cas à l'application des règles de domanialité publique, régime particulier de propriété publique. Le non-respect des dispositions propres au déclassement, en l'espèce l'article L. 451-5 du Code du patrimoine, ne pouvaient alors être invoquées. Le juge, cependant, ne suit pas l'argument, considérant que l'article 16-1 du Code civil n'a « ni pour objet ni pour effet de faire obstacle à l'exercice d'un régime de domanialité publique sur un reste humain » en application des dispositions du Code du patrimoine. Ainsi la Ville de Rouen n'était-elle pas fondée à restituer «ce bien sans respecter la procédure de déclassement prévue par l'article L. 451-5 du Code du patrimoine ». Les restes humains peuvent être un objet de propriété, y compris entre les mains d'une institution telle un musée $\mathrm{e}^{10}$. Pour autant, leur condition de bien est particulière, notamment quant à leur capacité à circuler dans le commerce. Le Code civil prohibe tout droit patrimonial. Il faut s'entendre sur cette formule quelque peu ambiguë.

\section{La prohibition d'un droit patrimonial}

«Le corps humain, ses éléments et ses produits ne peuvent faire l'objet d'un droit patrimonial » selon l'article 16-1 du Code civil et l'article 16-5 précise encore que « les conventions ayant pour effet de conférer une valeur patrimoniale au corps humain, à ses éléments ou à ses produits sont nulles ». C'est entre autres cet argument qu'avait avancé la Ville de Rouen pour dénier tout statut de propriété publique à la tête maorie présente dans ses collections. Mais c'était se méprendre sur le sens de la formule. La prohibition de tout droit patrimonial introduite dans le Code civil par les lois dites bioéthiques en 1994 avait simplement pour objet d'interdire tout commerce lucratif ${ }^{11}$. Le corps humain ne peut faire l'objet de transactions marchandes. Il s'agissait là d'instituer une forme d'extracommercialité du corps humain, non d'en déclarer l'extrapatrimonialité. En ce sens, la référence aux notions de droit patrimonial et de valeur patrimoniale n'était guère heureuse, introduisant une confusion sur l'interprétation du texte ${ }^{12}$. Cette prohibition emporte plusieurs conséquences pour les institutions publiques qui ont vocation à conserver ces pièces. Cela signifie qu'elles ne peuvent, sur le marché de l'art, acquérir des éléments du corps humain. En l'absence de jurisprudence sur ce point, la portée de la prohibition ne s'impose cependant pas de façon claire. Est-elle absolue et concernet-elle tout reste humain, y compris très ancien, une momie par exemple, ou des pièces archéologiques? On pourrait bien considérer qu'elle ne vise que les seuls éléments dont le commerce serait susceptible de heurter le principe de dignité humaine. Mais en réalité, cette borne est, aujourd'hui, très incertaine. Tout dépend dans quel sens ce principe est interprété, s'il réserve le respect des familles ou des proches ou si plus largement, il est censé préserver la charge d'humanité que recèle le corps mort, que certains désignent comme une chose publique humaine ${ }^{13}$. C'est l'opinion que défendent certains auteurs ${ }^{14}$ et qui s'inscrit dans un mouvement très net d'objectivation du principe de dignité dans la jurisprudence ${ }^{15}$. Sous cette approche, cela veut dire que, par exemple, des corps ou restes anciens sans généalogie identifiée ne pourraient accéder au marché. La formule énergique des articles 16-1 et 16-1-1 semble bien militer en ce sens. Ce qui est sûr, c'est que certaines collections telles que des têtes réduites ou des urnes funéraires aujourd'hui proposées par des sociétés de vente ne devraient pas se retrouver sur le marché de l'art. 
Le Conseil des ventes volontaires suggère de distinguer, parmi les restes humains, ceux qui pourraient recevoir la qualification de biens culturels, seuls ces derniers pouvant être vendus. Le rapport d'activité de 2013 précise que «sauf lorsqu'ils constituent sans équivoque des biens culturels, l'opérateur de ventes volontaires s'abstient de présenter à la vente tout ou partie de corps ou de restes humains ou tout objet composé à partir de corps ou de restes humains ». Mais l'on peut rester très réservé sur cette analyse dès lors que le Code civil, de son côté, ne fait pas cette distinction ${ }^{16}$.

Si les musées ou autres institutions publiques, comme toute autre personne physique ou morale, ne peuvent acheter des restes humains, rien ne s'oppose à ce qu'ils conservent la propriété de leurs biens et qu'ils en acquièrent par la voie de libéralités (dons ou legs). Reste à se demander quelles contraintes implique cette fonction de conservation et, plus généralement, en quoi le statut spécifique des restes humains influence les missions du musée. C'est la question de la patrimonialité des biens cette fois-ci regardée d'un point de vue culturel.

\section{La patrimonialité au sens culturel du terme : le cadre d'exercice des missions muséales}

Le corps, passant au travers du dispositif de l'après-décès tel que réglé par les lois funéraires, se trouve, dans la réalité des faits, conservé et exposé dans des espaces divers : musées des beaux-arts, musées d'histoires, muséums, musées d'universités, conservatoires, etc., et sous des formes multiples. Le questionnaire adressé, au nom du groupe de travail sur les restes humains piloté par M. Van Praët, aux institutions susceptibles de posséder ce type de pièces en dénombre sous de multiples catégories : pièces anatomiques et anthropologie physique française et étrangère, pièces archéologiques françaises et étrangères, momies amérindiennes, égyptiennes, reliquaires, etc. ${ }^{17}$ Le système français reconnaît ces pièces comme éléments du patrimoine culturel cependant que leur statut est influencé par le principe de dignité.

\section{Les restes humains, objets d'une servitude culturelle}

L'intérêt public qui motive une protection des restes humains fondée sur un dispositif du Code du patrimoine peut être de plusieurs sortes : intérêt scientifique, archéologique, documentaire, ce sont des archives du sol. Le registre est parfois aussi celui de l'esthétique. On songe aux préparations anatomiques de Fragonard exposées au musée de l'École nationale vétérinaire d'Alfort (MEVA), à Maisons-Alfort (fig. 1). Rien ne s'oppose, dans les textes, à ce qu'un reste humain, répondant à ce critère de l'intérêt public puisse faire l'objet d'une mesure de protection ${ }^{18}$. La collection du conservatoire d'anatomie de la faculté de médecine de Montpellier a, par exemple, été classée au titre des Monuments historiques. De la même façon, l'intégration de ce type de pièces dans une collection sous label musée de France est juridiquement possible, au regard de la définition de la collection définie comme un ensemble de biens qui revêtent un intérêt public du point de vue de leur conservation et de leur présentation. Les dispositions du Code civil ne font pas échec au processus de patrimonialisation ainsi engagé. C'est ce qu'a rappelé le juge dans l'affaire de la tête maorie du musée de Rouen. Selon la Ville de Rouen, propriétaire du musée, « le principe de la dignité de la personne humaine qui prolonge par-delà la mort les droits du défunt sur son corps (s'opposait) à ce que des éléments du corps humain puissent être classés comme éléments des collections des musées de France ", les dispositions de l'article 16-1 du Code civil l'emportant sur celles du Code du patrimoine. Le juge au contraire décide que les dispositions du Code du patrimoine, «qui rendent inaliénables les biens d'une personne publique constituant une collection des musées de France, placent ces biens sous un régime de protection particulière distinct du droit patrimonial énoncé à l'article 16-1 du Code civil ». L'idée est que, dans la mise en regard des ressorts du droit civil et du droit public, la protection patrimoniale n'est pas incompatible avec le respect dû au corps humain. Et l'on peut admettre que la nature de l'activité muséale ou encore le type de protection tiré du classement au titre des Monuments historiques ne heurte pas le principe de dignité. D’une certaine façon, rejoignant le patrimoine collectif sous la garde et la surveillance de l'État, le statut des restes humains est en concordance avec leur condition de « chose publique humaine».

Il peut cependant arriver qu'en dépit de la persistance d'un intérêt public attaché à leur protection, le législateur décide de faire prévaloir le principe de dignité. C'est l'objet de la loi de 2010, concentrée sur la restitution à la NouvelleZélande des têtes maories ${ }^{19}$. Il n'est pas exclu que d'autres pièces de ce même type connaissent un sort identique ${ }^{20}$. Dans un état des discussions autour du projet de loi sur la liberté de création, l'architecture et le patrimoine, il avait été question de favoriser les restitutions volontaires de restes humains, mais la loi finalement adoptée n'en a pas retenu le principe.

Si en soi et sous les réserves qui viennent d'être indiquées, un dispositif de protection est licite au regard du principe de dignité, reste à se demander dans quelles limites s'exercent les missions «patrimoniales ». La question s'est posée d'une façon plus aigüe à propos de l'exposition des restes humains.

\section{Les restes humains, sujets de dignité}

Si le fait de détenir, dans les collections publiques, ou de protéger des restes humains est compatible avec le statut des restes humains, il faut se demander dans quelles limites s'exercent les missions attachées à leur sauvegarde. C'est notamment à propos de la fonction d'exposition que la question du respect du principe de dignité s'est trouvée posée dans l'enceinte judiciaire. L'exposition de cadavres plastinés accueillie à la Pinacothèque de Paris en 2009 a généré un 
contentieux révélateur des difficultés qu'éprouve le juge dans l'appréhension du cadavre comme sujet de dignité21. Saisi d'une demande de fermeture de l'exposition, le juge des référés invoque des motifs tirés de l'esthétique discutable de l'événement, de la mise en scène dite "déréalisante » qu'il estime alors contraire à une visée scientifique ${ }^{22}$. La Cour d'appel développe un autre discours, sans doute conscient que le raisonnement du premier juge peut mettre en cause la conservation de certaines pièces dans les musées. Il indique que « le respect n'interdit pas le regard de la société sur la mort, et sur les rites religieux ou non qui l'entourent dans les différentes cultures, ce qui permet de donner à voir aux visiteurs d'un musée des momies extraites de leur sépulture, voire d'exposer des reliques sans entraîner d'indignation ni de trouble à l'ordre public ${ }^{23}$ ». La cour d'appel se concentre sur la question du défaut de consentement donné par les personnes pour prohiber l'exposition ${ }^{24}$. La Cour de cassation confirme la censure de l'exposition, mais recentre le propos sur la seule question de l'interdiction d'un droit patrimonial ${ }^{25}$. L'exposition incriminée est exclusivement réalisée à des fins lucratives, exploitation contraire au principe de dignité tel que prescrit dans les articles 16-1 et suivants du Code civil.
Les limites du droit se rapportant à ce critère de lucrativité, on le voit au travers de cet exemple, restent peu contraignantes pour le musée. Sous ce rapport, on peut être surpris de l'initiative du Comité consultatif national d'éthique. En 2010, il a édicté, en forme de norme générale, un certain nombre de préconisations, mettant en question les pratiques muséales d'exposition et leur utilisation à des fins scientifiques, se prononçant notamment sur l'illégitimité de la détention de ces restes ${ }^{26}$. Si l'on peut bien admettre que puisse se déployer une réflexion déontologique sur le « comment conserver, comment exposer », le cas échéant sur l'opportunité de restituer certaines pièces ${ }^{27}$, l'instauration de bonnes pratiques n'a pas vocation à créer du droit là où, précisément, le droit en a fixé les contours. Le fait est que le chantier est aujourd'hui en grande partie du côté de la pratique, avec de multiples questions ${ }^{28}$. Les problèmes dominants qui ressortent de l'enquête menée sur la situation des restes humains dans les musées et universités tiennent au désintérêt pour ce type de pièces notamment de la part des élus - ils sont souvent tentés de se défaire de ces éléments, voire de les supprimer ${ }^{29}-$ au déficit de documentation ainsi qu'à l'absence d'un personnel compétent. C'est pourquoi la réflexion collective sur les conditions de conservation, de restauration, d'études et de mise en valeur est indispensable.

\section{Notes}

1. Loi n 2008-1350 du 19 décembre 2008 relative à la législation funéraire qui, notamment, modifie le Code civil et le Code général des collectivités territoriales.

2. Pour reprendre l'expression de Puffendorf, sur la question du corps au musée, voir Cornu, 2009, p. 1907-1914.

3. L'article L. 1 s'est par ailleurs enrichi d'un second paragraphe introduit par la loi $\mathrm{n}^{\circ}$ 2016-925 du 7 juillet 2016 relative à la liberté de création, à l'architecture et au patrimoine aux termes duquel «Il s'entend également des éléments du patrimoine culturel immatériel, au sens de l'article 2 de la convention internationale pour la sauvegarde du patrimoine culturel immatériel, adoptée à Paris le 17 octobre 2003 ». Quoique la question des restes humains puisse recéler des aspects liés au patrimoine immatériel (les rites et pratiques religieuses et funéraires notamment), notre approche du sujet est ici concentrée sur le patrimoine tangible.

4. Cette perception du monde au travers de la division cardinale entre les personnes et les choses est ancienne dans le droit. Elle continue de structurer fortement le droit contemporain. Sur ce constat que le cadavre est pour le droit une chose et sur l'inévitable réification du cadavre humain, F. Bellivier, Droit des personnes, LGDJ, Lextenso éd., 2015, n 208, p.192.

5. Selon la formule de Yann Thomas, la valeur des choses. Le droit romain hors la religion, in Annales. Histoire, Sciences Sociales. $57^{\mathrm{e}}$ année, N. 6, 2002. p. 1431-1462, selon lequel le régime d'exception des choses inestimables fait comprendre, par antithèse, le droit ordinaire de toutes les autres choses. 6. Ces diverses qualifications ont pu être avancées soit par la doctrine, soit par la jurisprudence.

7. Même si l'atteinte à l'intégrité du cadavre dans le Code pénal relève de l'atteinte à la personne humaine (art. 22517 ), voilà qui montre bien l'inadéquation des catégories du droit en la matière.

8 . Notamment plusieurs jugements émanant du Tribunal de grande instance de Lille.

9. Idée renforcée par la législation funéraire de 2008 dont l'objet a aussi été de combattre toute forme de privatisation des cendres, en ce sens, F. Bellivier, op. cit., $\mathrm{n}^{\circ}$ 216. C'est selon la formule de F. Terré et P. Simmler, une propriété commune d'intérêt familial, in Le droit des biens, Précis Dalloz, n 772, p. 417.

10. Propriété qui céderait évidemment devant une revendication familiale car alors domineraient sans discussion les droits de la famille du défunt, v. CAA de Douai du 30 novembre 2006 qui, à propos de dépouilles de soldats de la Première Guerre mondiale trouvés sur un site pour lesquels les juges indiquent qu'en cas de réclamation des familles, les dépouilles doivent être mises à la disposition des familles. Elle peut aussi céder lorsqu'une loi impose la restitution, comme en 2002 s'agissant de la dépouille de la Vénus hottentote, $1 . n^{\circ}$ 2002-323 relative à la restitution par la France de la dépouille mortelle de Saartjie Baartman à l'Afrique du Sud, ou, en 2010, à propos des têtes maories détenues dans des musées de France qui ont fait l'objet d'une obligation de restitution, loi $\mathrm{n}^{\circ} 2010-501$ du 18 mai 2010.

11. Tout commerce au sens juridique du terme, dans la mesure où les dons sont autorisés et la donation est une forme de commerce, quand bien même elle se réalise sans contrepartie.

12. On peut mettre en parallèle l'expression de droit patrimonial utilisée dans le domaine du droit d'auteur, qui renvoie aux droits pécuniaires ou économiques de l'auteur par opposition à son droit moral.

13. Sur ce mouvement d'humanisation visible dans le droit contemporain en ce qui concerne les restes du cadavre, F. Bellivier, op. cit., $\mathrm{n}^{\circ} 219$, p. 202.

14. Notamment G. Loiseau à propos du nouvel article L.16-1-1 du Code civil et sur l'idée que la dépouille est de ces « choses mal logées dans le seul ordre des biens sans trouver refuge dans celui des personnes ", in Pour un droit des choses, Dalloz, 2006, ${ }^{\circ} 44$, p. 3020.

15. En ce sens, Th. Pech, Réflexions sur la dignité de l'être humain en tant que concept juridique du droit français, Dalloz, 1997, chronique, p. 65.

16. Conformément à l'adage Ubi lex non distinguit, nec nos distinguere debemus, là où la loi ne distingue pas, il convient de ne pas distinguer.

17. La constitution de ce groupe de travail est une initiative conjointe du 
ministère de la Culture et du secrétariat d'État à l'Enseignement supérieur et à la recherche, créé en lien avec la commission scientifique nationale des collections. Piloté par M. Van Praët, il est chargé, spécialement, de réfléchir à cette question des collections des restes humains, d'en faire un état des lieux, d'où le questionnaire, d'étudier les aspects juridiques en France et à l'étranger et, notamment, le statut patrimonial de ces pièces présentes dans les collections, d'étudier la question des critères de sortie des collections en cas de revendication, de se pencher sur les conditions de gestion de ces éléments. Un premier texte a été publié dans le rapport remis par la CSNC, disponible en ligne.

18. Même si la pratique des services concernés reste réservée sur la mise en mouvement de ce type de protection en matière de restes humains.

19. Animée par le même esprit, la loi n 2002-323 du 6 mars 2002 avait également ordonné la restitution de la dépouille de Saartjie Baartman à l'Afrique du Sud.

20. Il n'existe pas, actuellement, de texte contraignant sur cette question de la restitution des restes humains au plan international, du moins d'un point de vue spécifique. On peut certes évoquer la résolution adoptée par l'Assemblée générale de l'ONU le 13 septembre 2007 sur les droits des peuples autochtones qui demande qu'une « réparation » soit apportée en cas d'atteintes à leur patrimoine culturel et religieux et reconnaît " un droit au rapatriement de leurs restes humains », invitant les États à permettre «l'accès aux objets de culte et aux restes humains en leur possession et/ou leur rapatriement par le biais de mécanismes justes, transparents et efficaces mis au point en concertation avec les peuples autochtones concernés » (Déclaration des Nations unies sur les droits des peuples autochtones, adoptée par l'Assemblée générale le 13 septembre 2007, article 12). Mais il n'emporte pas d'obligations positives de restitution à la charge des États.
21. Exposition Our Body, à corps ouver inspirée des préparations de l'anatomiste Gunther Von Hagens.

22. Ordonnance de référé rendue par le Tribunal de grande instance de Paris, 21 avril 2009, n0 09/53100, AJDA, 2009, p. 797.

23. Cour d'appel de Paris, 30 avril 2009, n 09/09315, Dalloz, 2009, p. 2019.

24. Argument dont on peut discuter la pertinence dès lors que la destination du corps est prévue et imposée par la loi.

25. Cour de cassation, 16 septembre 2010, arrêt $\mathrm{n}^{\circ} 764$.

26. Évoquant notamment la Déclaration des Nations unies sur les droits des peuples d'origine adoptée par l'Assemblée générale du 13 septembre 2007 avec laquelle la France serait en contradiction si elle refusait de faire droit aux demandes de peuples concernés. Avis n 111 sur les problèmes éthiques posés par l'utilisation des cadavres à des fins de conservation ou d'exposition muséale de J.-C. Ameisen, P. Le Coz, Comité Consultatif National d'Ethique pour les Sciences de la vie et de la santé, Paris, le 7 janvier 2010.

27. On peut évoquer ici les dispositions contenues dans le Code d'éthique de l'ICOM, pour lequel les musées se doivent de « répondre avec diligence, respect et sensibilité aux demandes de retrait, par la communauté d'origine, de restes humains ou d'objets à portée rituelle exposés au public », envisageant la restitution de biens culturels exportés de manière illicite ou bien faisant « partie du patrimoine culturel ou naturel du pays ou de la communauté qui demande leur retour ", Code de déontologie de l'ICOM pour les musées, 2006, § 4.4 (cf. aussi $§ 2.5,3.7$ et 4.3).

28. En l'occurrence, un certain nombre de rencontres ont été consacrées à l'étude de cette question en présence de conservateurs et de restaurateurs, particulièrement dans le cadre de l'INP.

29. De ce point de vue, la question de l'archéologie a été identifiée comme un vrai sujet.

\section{Bibliographie}

Baud J-P., 1993, L'affaire de la main volée, une histoire juridique du corps, Paris, éditions du Seuil.

Bellivier F., 2015, Droit des personnes, LGDJ, Paris, éditions Lextenso.

Code de déontologie de l'ICOM pour les musées, 2006.

Cornu M., 2009, « Le corps humain au musée, de la personne à la chose ", Recueil Dalloz, p. 1907-1914.

Doucet J.-P., 1979, La condition juridique du cadavre, Liège.

Fenouillet D., 1997, « Respect et protection du corps humain, article 16 à 16-2 du Code civil ", Ed. du Juris-classeur, fasc. $12, \mathrm{n}^{\circ} 56$.

Gridel J.-P., « $\mathrm{V}^{\circ}$ Cadavre », in M. Marzano (dir.), Dictionnaire du corps, Paris, PUF.

Hermitte M.-A., 1988, « Le corps hors commerce, hors marché », APD, tome XXXIII, Paris, Sirey.

Labbée X., 2002, «La personne, l'âme et le corps », $L P A$; note sous TGI Lille 10 novembre 2004, « La valeur des choses sacrées ou le prix des restes mortels », Dalloz.

Loiseau G., 2006, «Pour un droit des choses ", Dalloz, n 44, p. $3020 ; 2009$, "Mortuorum corpus, une loi pour le respect », Dalloz, n 4, p. 236.

Marin J-Y., 2006, «Statut des restes humains, revendications internationales ", Le patrimoine culturel religieux, enjeux juridiques et pratiques cultuelles, L'Harmattan, p. 337.

Négri V., 2006, «Fouilles archéologiques et questions de religion ", Le patrimoine culturel religieux, L'Harmattan, coll. droit du patrimoine culturel et naturel.

Pech Th., 1997, «Réflexions sur la dignité de l'être humain en tant que concept juridique du droit français ", Dalloz, chronique, p. 65.

Raimbault Ph., 2005, « Le corps humain après la mort, quand les juristes jouent au cadavre exquis ", Droit et société 61 , p. 817 et s.

Thomas Y., 2002, « La valeur des choses. Le droit romain hors la religion ", in Annales. Histoire, Sciences Sociales, $57^{\mathrm{e}}$ année, N. 6, p. 1431-1462. 